\title{
BMJ Open Correlation of impulsivity with self- harm and suicidal attempt: a community study of adolescents in Taiwan
}

\author{
Yu-Hsin Huang, ${ }^{1,2,3,4}$ Hui-Ching Liu, ${ }^{2,3,4}$ Fang-Ju Tsai, ${ }^{5,6,7}$ Fang-Ju Sun, ${ }^{4,8}$ \\ Kuo-Yang Huang, ${ }^{9}$ Yu-Ching Chiu, ${ }^{10}$ Yen-Hsun Huang, ${ }^{11}$ Yo-Ping Huang, ${ }^{12}$ \\ Shen-Ing Liu ${ }^{1,2,3,4,8}$
}

To cite: Huang Y-H, Liu H-C, Tsai F-J, et al. Correlation of impulsivity with self-harm and suicidal attempt: a community study of adolescents in Taiwan. BMJ Open 2017;7:e017949. doi:10.1136/ bmjopen-2017-017949

- Prepublication history for this paper is available online. To view these files, please visit the journal online (http://dx.doi. org/10.1136/bmjopen-2017017949).

Received 30 May 2017 Revised 19 0ctober 2017 Accepted 23 October 2017

CrossMark

For numbered affiliations see end of article.

Correspondence to

Dr Shen-Ing Liu;

maryliuyip@gmail.com

\section{ABSTRACT}

Objectives The aim of this study was to investigate differences and similarities in risk factors for deliberate self-harm (DSH) and suicidal attempt (SA), and the role of impulsivity among a group of community adolescents.

Setting This is a cross-sectional study conducted at high schools in Northern Taiwan.

Data and participants We recruited grade 1 students from 14 high schools. A total of 5879 participants (mean age 16.02 years, female adolescents: $57.7 \%$ ) completed the online assessment.

Outcome measures Participants completed online questionnaires about sociodemographic data, suicidality, history of DSH and SA, depressed mood, self-esteem, social support, family discord, impulsivity (Barratt Impulsiveness Scale Version 11 (BIS-11)) and the use of alcohol, tobacco and illicit drugs. A subsample was interviewed about lifetime SA, and the results were compared with those from the online questionnaires.

Results In our sample, $25 \%$ of the students had lifetime DSH and $3.5 \%$ had lifetime SA. Two hundred and seventy-two students received face-to-face interviews. The concordance between the online questionnaires and interviews in terms of ascertaining cases of SA was moderate (concordance rate $82.76 \%$; kappa value 0.59 ). Similar risk factors for DSH/SA among the whole sample included female gender, lower academic performance, depression, substance use (tobacco and alcohol) and low self-esteem. The BIS-11 score was correlated with DSH. Factor 3 score of the BIS-11 (novelty seeking) was correlated with DSH in both boys and girls, whereas factor 2 score (lack of self-control) was correlated with $\mathrm{SA}$ in boys. Social support was a protective factor against SA among the female adolescents. Gender modulated the association of impulsivity and DSH/SA. Associations between impulsivity and DSH and SA were particularly strong among boys.

Conclusions Risk factors for DSH and SA were similar, but not identical. Early identification of those at risk and appropriate interventions may be helpful.

\section{INTRODUCTION}

According to the WHO, suicide was the second-leading cause of death worldwide in 2012 among people aged 15-29 years. ${ }^{1}$ In addition, a systemic review reported a

\section{Strengths and limitations of this study}

- This study used a large community sample of adolescents from different areas of Taipei, which is a strength when it comes to generalisation.

- A further strength of the present study is that wellestablished self-reported measures and a broad array of relevant psychosocial measures were used.

- Few studies based in Asian countries have investigated the relationship between impulsivity and deliberate self-harm (DSH) and suicidal attempt (SA) in a community sample.

- The agreement between online assessments and interviews with regards to lifetime SA was moderate, which showed that an online study is a reliable way to investigate suicidality.

- Gender is a moderator between the relationship of impulsivity and adolescent DSH/SA.

strong association between self-harm and later suicide, with the risk of suicide among patients with deliberate self-harm (DSH) being hundreds of times higher than that in the general population. ${ }^{2}$ The terminology surrounding DSH is, however, complex. In this study, we defined DSH as self-harmful behaviour, regardless of an individual's intention to die. ${ }^{3}$ The following behaviours are examples of DSH: initiating a behaviour (eg, self-cutting, ${ }^{3}$ jumping from a height, ${ }^{4}$ burning $^{5}$ and hanging ${ }^{5)}$ intended to cause self-harm; ingesting a substance in excess of the prescribed or generally recognised therapeutic dose $^{3-5}$; ingesting a recreational or illicit drug that was an act that the person regarded as being self-harm ${ }^{45}$; and ingesting a non-ingestible substance or object. ${ }^{4}$

Suicidal attempt (SA), that is, self-harm with the intent to die, is less common than DSH. The estimated lifetime prevalence of SA among adolescents ranges from $3.0 \%$ to $8.4 \%{ }^{6-8}$ compared with $4.0 \%-30 \%$ for $\mathrm{DSH}^{8}{ }^{8}$ Despite increased awareness and research on DSH and SA among adolescents, few studies 
have investigated the extent to which risk factors for DSH and SA overlap in a community setting, especially in Asian countries.

Regarding aetiology, there is a biopsychosocial model for self-harm. Higher levels of endogenous opioids and higher level of pain tolerance have been found in selfharming people. Interactions between environmental and genetic factors may also contribute to self-harm. ${ }^{9}$ There is also a stress-diathesis or a psychobiological model for SA. ${ }^{10}$ Deficits in serotonergic neurotransmission, low cerebrospinal fluid 5-hydroxyindoleacetic acid (5-HIAA) levels, low platelet 5-hydroxytryptamine and a decreased number of binding sites in platelets have been found in people with SA. ${ }^{11}$ A potential link between low plasma 5-HIAA levels and impulsivity/severity of the SA has also been postulated. ${ }^{11}$ Different aetiologies may differentiate between DSH and SA, and we were also interested in the role of impulsivity in these two groups.

Impulsivity has frequently been reported to be a risk factor for both DSH and SA, ${ }^{4}$ although the results are somewhat inconsistent. In one study, the association with SA was non-significant after controlling for hopelessness, neuroticism, external locus of control, self-esteem and extroversion. ${ }^{13}$ In addition, Hawton et al ${ }^{14}$ found that impulsivity was an independent risk factor for self-harm among adolescent girls but not among adolescent boys. In contrast, another study in Ireland reported that impulsivity was a risk factor for DSH among boys but not girls. ${ }^{15}$ These inconsistent findings imply that impulsivity may be important in identifying high-risk subgroups. To date, most previous studies on the relationship among impulsivity, DSH and SA in adolescents have been conducted in Western countries.

Due to these inconsistent results and to bridge the gaps in current knowledge, we designed this study to investigate correlations among impulsivity, DSH and SA in Taiwanese adolescents. The study aimed to: (1) explore possible differences and similarities in risk factors among adolescents with DSH and SA and (2) explore the role of impulsivity in these two groups.

\section{METHODS \\ Subjects}

It was part of the Taiwanese Adolescent Self-Harm Project (TASP), a prospective study conducted to evaluate the 1-year incidence of self-harm behaviour and the associated risk factors among adolescents in Taiwan. ${ }^{16}$ From October 2008 to March 2010, we recruited firstgrade students from 14 senior high schools in Taipei and New Taipei City, Taiwan by purposive sampling. The participating schools were chosen from different regions of Taipei, including urban, suburban and rural areas, and accounted for $11.7 \%$ of all high schools in the Taipei area. After the aims of this study had been fully explained to both the students and their parents, written informed consent was obtained from all parents and participants.

\section{Online assessments}

All of the participants completed the Chinese version of the Multidimensional Scale of Perceived Social Support (MDSS),${ }^{17}$ the Chinese version of the Patient Health Questionnaire (PHQ-9), ${ }^{18}$ the Chinese version of the Barratt Impulsiveness Scale Version 11 (BIS-11) ${ }^{19}$ and the Rosenberg Self-Esteem Scale (RSES).$^{20}$ The students also reported all self-harm experiences, physical illnesses and substance use. All of the measures were completed anonymously online at the participating schools, and a trained research assistant was also available. A subsample of the students also received diagnostic interviews conducted by child psychiatrists. ${ }^{18}$ The data from the online questionnaires were collected by a computer engineer. The researchers had access to the data 1 day after the students had completed the questionnaires to ensure the quality of the data. We also provided each school with a list of students at high risk of suicide for further referral and on-site counselling by a child psychiatrist or a psychologist at their school.

\section{Suicidality: suicidal ideation, suicidal plan, DSH and SA}

All subjects were asked if they had ever thought of killing themselves at any time in their life. They were then asked about ever having planned to kill themselves. Using the same scale, the students were then asked 'have you ever deliberately (not accidentally) hurt yourself?' and 'how many times have you deliberately self-harmed?' The participants who responded positively to the main questions were then asked to elaborate on their actions (multiple choices including drug overdose, hanging, burning charcoal, jumping from a height, cutting themselves and being hit by a car). They were then asked to describe the act, the number of episodes, the timing of each episode and the consequences (eg, need for medical intervention) and to endorse the motive behind the act. SAs were identified in all reports of self-harm according to the response to, 'have you ever really tried to kill yourself during these DSH episodes?' We also collected the number of SAs and the time of the first and last attempts. The subjects who reported having harmed themselves with/without suicidal intent were classified as 'DSH'. SA was defined as an intentional action to kill oneself.

\section{Chinese version of the BIS-11}

The BIS-11 is a 30-item self-reported questionnaire designed to measure impulsivity. The total score ranges from 30 to 120 , with a higher score indicating greater impulsivity. ${ }^{21}$ The 25 items of the Chinese version of the BIS-11 have demonstrated good overall internal consistency in Taiwanese adolescents, with a Cronbach's $\alpha$ of $0.834{ }^{19}$ The factor structure of the Chinese version of the BIS-11 consists of three factors: 'inability to plan', 'lack of self-control' and 'novelty seeking'.

\section{Multidimensional Scale of Perceived Social Support}

The MDSS is a self-reported measure of the availability and adequacy of social support from various sources. ${ }^{17} \mathrm{We}$ 
estimated four types of social support, including that from parents, other family members, friends and teachers, with a higher score indicating greater social support. In our sample, the Cronbach's $\alpha$ was 0.63 .

\section{Chinese version of the PHQ-9}

The PHQ-9 consists of nine items evaluating the presence of one of the nine Diagnostic and Statistical Manual of Mental Disorders (Fourth edition) (DSM-IV) criteria of major depressive episodes during the past 2 weeks. The total score ranges from 0 to 27, with higher scores indicating an increased likelihood of major depressive disorder (MDD). The Chinese version of the PHQ-9 has been validated for detecting MDD and has shown good internal consistency $(\alpha=0.84)$ and acceptable test-retest reliability (intraclass correlation coefficient $=0.80$ ) among adolescents in the community. ${ }^{18} \mathrm{~A}$ PHQ-9 score $\geq 15$ had a sensitivity of 0.72 and a specificity of 0.95 for recognising MDD.

\section{Chinese version of the RSES}

The RSES consists of 10 items that refer to self-respect and self-acceptance. ${ }^{22}$ The Chinese version of the RSES has demonstrated acceptable internal consistency. The reliability and validity of the Chinese version of the RSES have also been demonstrated. ${ }^{20}$

\section{Substance use variables}

Data on substance use included information on cigarette smoking, high-risk alcohol use and any illicit drug use. The participants who reported that they smoked currently were classified as current cigarette smokers. High-risk alcohol use was assessed using the Chinese version of the Alcohol Use Disorders Identification Test-Consumption (AUDITC). The AUDIT-C includes the first three questions from the full AUDIT ${ }^{23}$ and assesses the amount and frequency of alcohol intake and frequency of alcohol misuse (defined as having six or more drinks). The optimal cut-off score of the Chinese AUDIT-C for hazardous drinking was threefourths, with good sensitivity (0.90) and specificity (0.92) ${ }^{24}$ In this study, this was treated as a continuous variable, and a higher score indicated more alcohol use. A summary of illicit drug use was obtained from the participants who had used any form of illicit drug during the past month.

\section{Face-to-face interviews with child psychiatrists using the K-SADS-E}

The Kiddie-Schedule for Affective Disorder and Schizophrenia Epidemiological Version (K-SADS-E) is a semistructured interview scale for the systematic assessment of both past and current episodes of psychiatric disorders in children and adolescents. The Chinese version has been shown to be a reliable and valid instrument. ${ }^{25}$ Child psychiatrists blind to the results of the online assessments interviewed a subsample of the students $(n=272)$ using the K-SADS-E. After 12 months of the initial assessment, the students completed the same questionnaires. All of the students with new occurrences of self-harm in the past year (ie, the students who did not report self-harm at entry but did the next year) were enrolled for face-to-face interviews. The students who did not report any occurrences of self-harm at both years were randomly selected on a 1:1 ratio, frequency matched by class and gender. For the students who reported occurrences of self-harm in both years, one in two received face-to-face interviews. The response rate was $94.8 \%$. Lifetime SA was assessed by the child psychiatrists according to the question, 'have you actually tried to kill yourself?'

\section{Statistical analysis}

Descriptive and analytical statistics of the data obtained in this study were analysed using SPSS V.21.0 for Windows (SPSS, IBM). Risk factors for DSH and SA were first analysed using univariate logistic regression analysis, with one variable at a time. Hierarchical multiple regression strategies (using a stepwise method) were then used to determine the risk factors for $\mathrm{DSH} / \mathrm{SA}$ and to assess whether impulsivity played any role. The joint effect of the independent variables that showed significance in univariate analysis was assessed with hierarchical regression analysis. To examine the association between impulsivity and DSH/SA, all sociodemographic and personality (BIS-11 and RSES) variables were introduced in the first step (model 1), followed by adding social support (MDSS) in the second step (model 2), alcohol/tobacco use in the third step (model 3) and depressive symptoms in the fourth step (model 4). We then examined the interaction between gender and BIS-11 among logistic regression analyses to see whether the relationship between impulsivity and DSH/SA was moderated by gender (model $5) .{ }^{26}$ Logistic regression was used to analyse the role of impulsivity and all other sociodemographic, clinical and personality trait variables with DSH/SA between genders (model 6). We further analysed three subscales of the BIS-11 instead of the total score in the male and female students.

We also examined the agreement (ie, reliability) between the computer questionnaire assessments and face-to-face interviews $(\mathrm{n}=272)$ with regards lifetime SA assessed at the second year. This agreement was assessed by concordance rate and kappa statistics. ${ }^{27}$

\section{RESULTS}

Descriptive statistics and univariate analysis of lifetime DSH and lifetime $S A$

We recruited 5879 students with a mean age of 16.02 years (SD 0.52). The overall response rate was $60.61 \%$. Table 1 shows the sociodemographic data of the students along with scores on the MDSS, PHQ-9, BIS-11, RSES and AUDIT-C. Twenty-five per cent of the students $(n=1472)$ reported self-harm behaviour, with a mean of 4.06 times (SD 3.33). Two hundred and six students (3.5\%) reported that their self-harm behaviour was actually a SA. The mean BIS-11 score was 62.9 for the students with lifetime DSH and 64.2 for those with lifetime SA. 


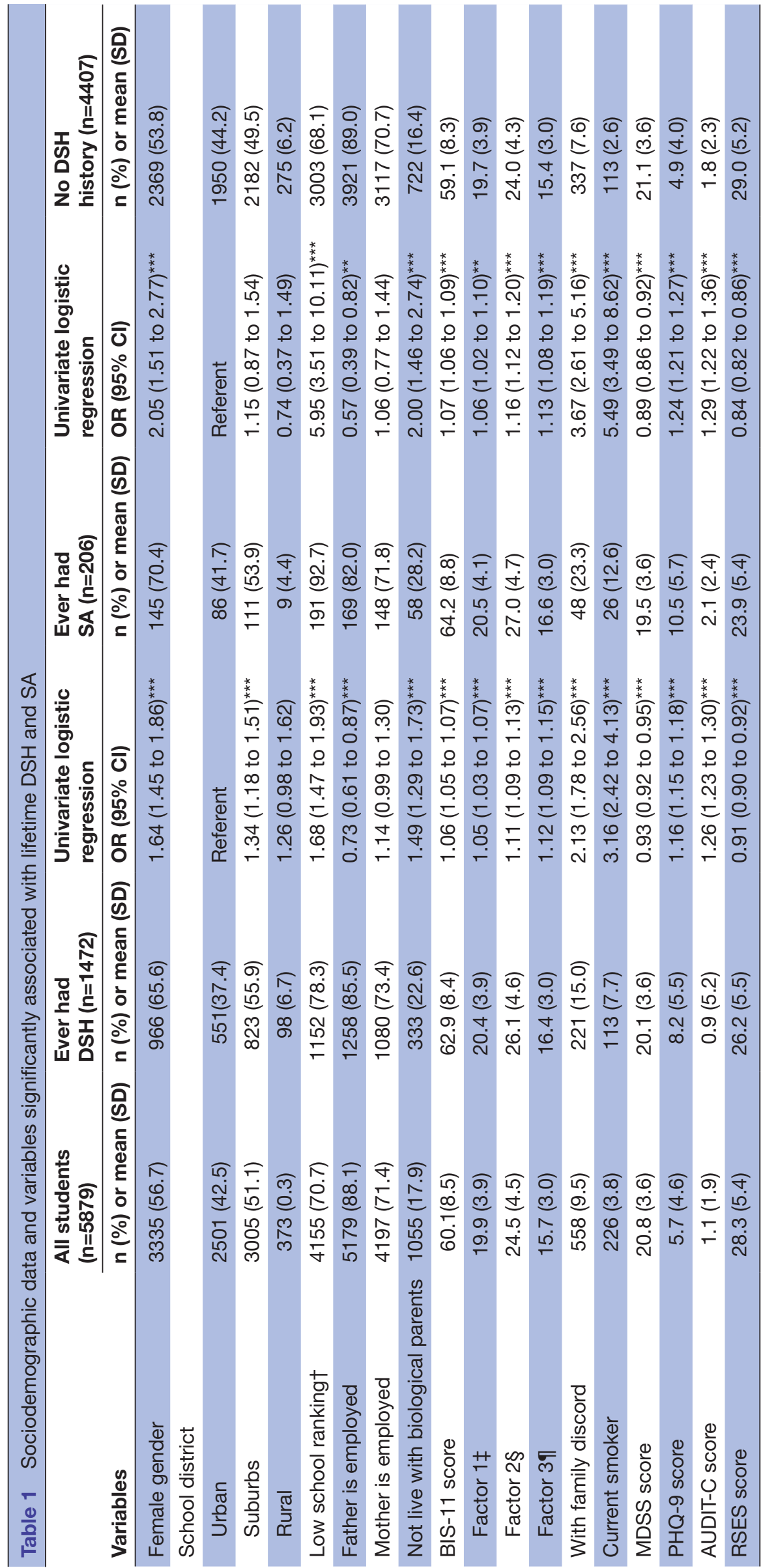

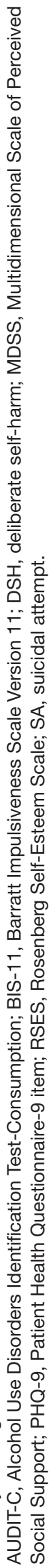


There was no statistical difference in BIS-11 score between the male and female students. Univariate analysis revealed that the risk factors associated with both lifetime DSH and SA were higher impulsivity, female gender, low school ranking, father's job status, not living with biological parents, family discord, low self-esteem, poor social support, currently smoking, more alcohol use and more depressive symptoms (table 1). Students in schools located in suburban areas compared with those in urban area had a higher risk of DSH.

\section{Hierarchical regression of lifetime DSH and lifetime SA}

The results of the hierarchical approach are presented in tables 2 and 3. The adjusted model 1 including sociodemographic variables and personality traits (RSES and BIS-11) showed that similar risk factors were associated with both DSH and SA. These included high impulsivity, low self-esteem, female gender, family discord and low school ranking. The factors of father's job status, school district and living with one's biological parents ceased to be significant predictors, indicating that these variables were fully statistically mediated by factors of gender, self-esteem, impulsivity, family discord and school ranking. In an unadjusted model, as the impulsivity score (BIS) increased by 1 unit, the likelihood of reporting a lifetime DSH and SA increased by approximately $6 \%$ and $7 \%$, respectively. The addition of gender, school district, family discord, school ranking and self-esteem factors attenuated the effect of impulsivity on the OR (1.04) (tables 2 and 3).

We then added social support status (MDSS score) into model 2. Social support did not significantly alter the confidence intervals of the other variables in model 1 with regards to DSH, and it was also not significantly associated with DSH in model 2. However, social support was a protective factor for SA in adjusted model 2. Impulsivity, female gender, low self-esteem, low school ranking and family discord retained significance in adjusted model 2 for SA.

In model 3, we added variables of substance use, and DSH and SA were still significantly associated with impulsivity, although the odds were attenuated (tables 2 and 3 ). The other significant variables in model 2 persisted as risk factors for DSH/SA in model 3. Currently smoking and a higher level of alcohol use were strong risk factors for $\mathrm{DSH} / \mathrm{SA}$.

In model 4, we included all variables (including depressive symptoms). DSH was still significantly associated with impulsivity, although its odds were attenuated again. The effect of family discord was fully statistically mediated in the final model of DSH. Other significant variables in model 3 persisted as risk factors for DSH in the final model. Depressive symptoms was a risk factor for DSH, and for every 1 unit increase in PHQ score, the likelihood of reporting lifetime DSH increased by $12 \%$. In addition, for every 1 unit increase in AUDIT-C score, the likelihood of reporting lifetime DSH increased by $15 \%$.

Impulsivity, family discord and social support lost their significance after adjusting for the aforementioned covariates in model 4 for the SA group (table 3). Depressive symptoms and substance use (alcohol and tobacco use) were stronger risk factors for SA than impulsivity. For every 1 unit increase in PHQ score, the likelihood of reporting lifetime SA increased by $19 \%$, and for every 1 unit increase in AUDIT-C score, the likelihood of reporting lifetime SA increased by $17 \%$. The effects of depression and alcohol use on SA was stronger than those on DSH. In addition, smoking had the strongest effect on SA, with current smokers having a threefold higher risk of SA compared with non-smokers.

When we added the interaction term of BIS-11 and gender in model 5 , we found that they were significant in both tables 2 and 3 . This meant that gender modulated the association between impulsivity and DSH/SA. Other risk factors besides gender remained significance in both tables 2 and 3.

We further analysed the risk factors for DSH/SA by gender in model 6 . For boys, impulsivity, low self-esteem, alcohol use and depressive symptoms were risk factors for DSH compared with low school ranking, low self-esteem, smoking, alcohol use and depressive symptoms in girls (table 2). The association between impulsivity and DSH was particularly strong among boys, whereas total impulsivity scores were not associated with SA in either gender. For boys, low self-esteem, smoking and depressive symptoms were risk factors for SA compared with low school ranking, low self-esteem, poor social support, smoking, alcohol use and depressive symptoms in girls, while their father's employment status was a protective factor (table 3). Smoking status was an especially strong risk factor for $\mathrm{SA}$ in girls $(\mathrm{OR}=5.24)$.

We used three subscales instead of BIS-11 total score in the multivariate logistic regression analysis to investigate the relationships among impulsivity subscales and DSH/SA (table 4). For both genders, factor 3 of the BIS-11 scale (novelty seeking and acting without thinking), low self-esteem, alcohol use and depressive symptoms were risk factors for DSH. Low school ranking and smoking were additional risk factors for DSH in girls. For boys, risk factors for SA included factor 2 of the BIS-11 scale (lack of perseverance and self-control), low self-esteem, smoking and depressive symptoms. For girls with SA, impulsivity subscales were non-significant.

\section{Agreement between online assessment and face-to-face interviews (concordance rate and kappa statistics)}

The concordance rate was the proportion of the same status of lifetime SA comparing the results of the online assessments and face-to-face interviews, which was $82.76 \%$ $((89+31) / 145)$ (table 5$)$. The kappa value was 0.59 , which was in the moderate agreement range. ${ }^{27}$ 


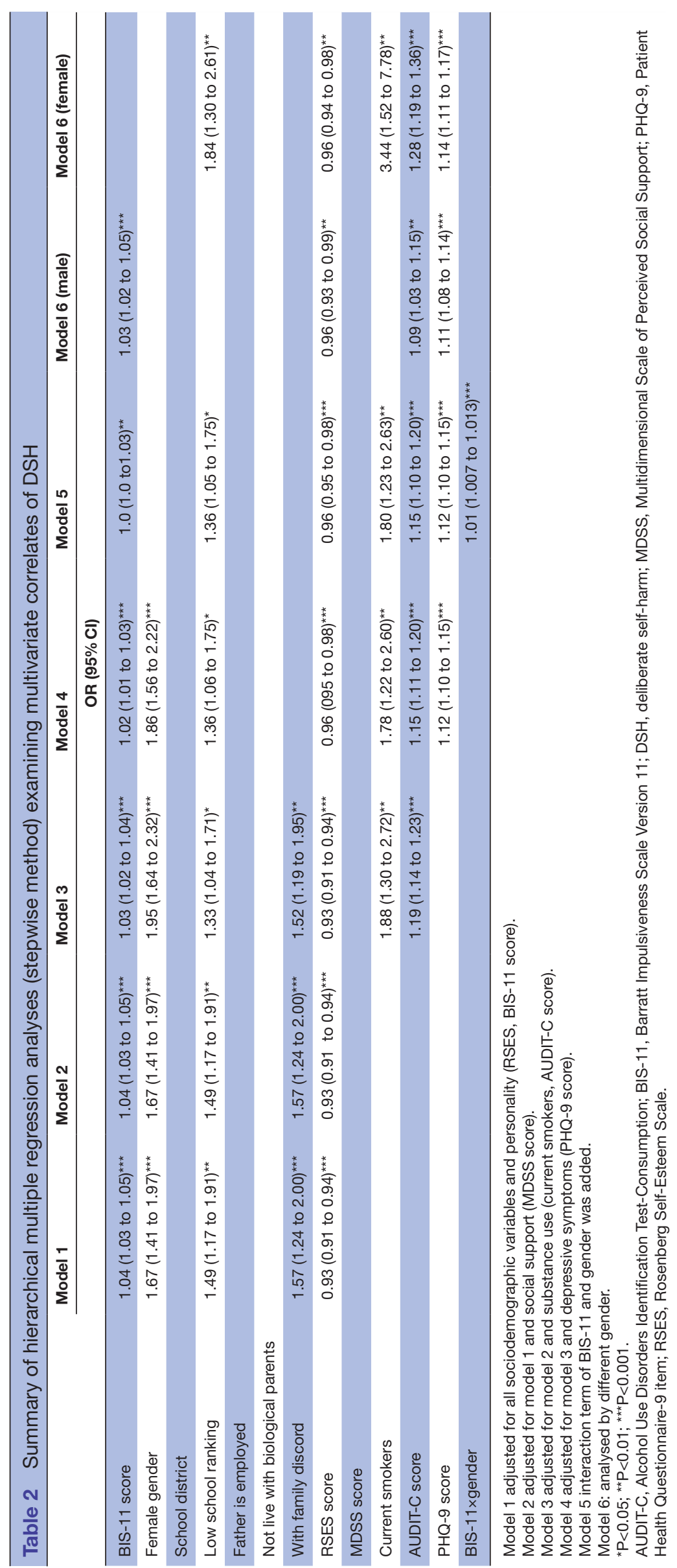

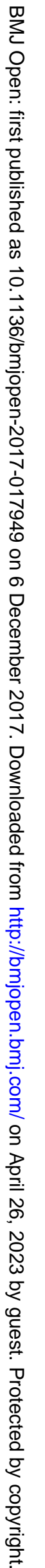




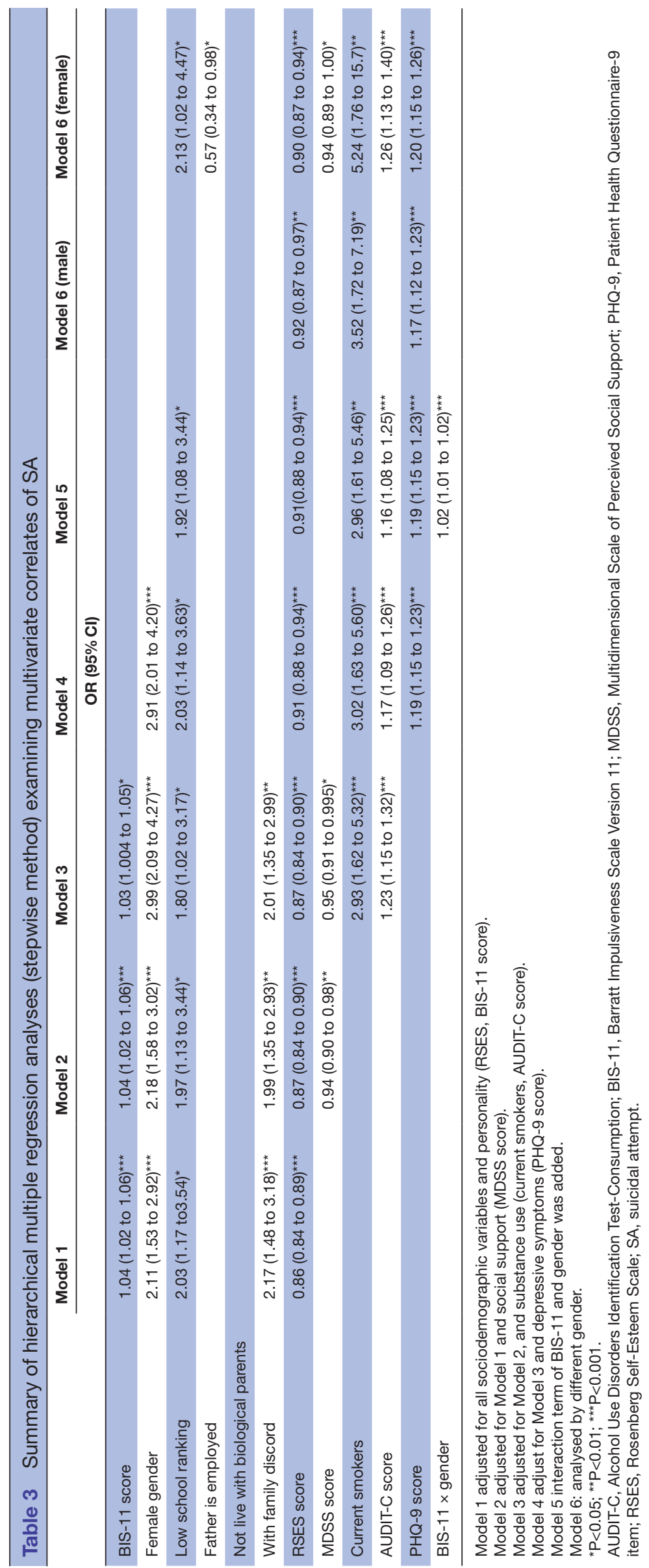


Table 4 Multiple regression analyses (stepwise method) examining multivariate correlates of DSH and SA, focusing on three subscales of the BIS-11

\begin{tabular}{lrrr} 
DSH (male) & DSH (female) & SA (male) & SA (female) \\
\hline & OR (95\% Cl)
\end{tabular}

\begin{tabular}{|c|c|c|c|c|}
\hline \multicolumn{5}{|l|}{ BIS-11 score factor $1 \dagger$} \\
\hline BIS-11 score factor $2 \ddagger$ & & & $1.07(1.01 \text { to } 1.14)^{\star}$ & \\
\hline BIS-11 score factor $3 \S$ & $1.10(1.05 \text { to } 1.16)^{\star \star \star}$ & $1.04(1.002 \text { to } 1.08)^{*}$ & & \\
\hline Father is employed & & & & $0.57(0.34 \text { to } 0.98)^{\star}$ \\
\hline \multicolumn{5}{|c|}{ Not live with biological parents } \\
\hline RSES score & $0.94(0.92 \text { to } 0.97)^{\star \star \star}$ & $0.96(0.93 \text { to } 0.98)^{\star \star \star}$ & $0.93(0.88 \text { to } 0.99)^{*}$ & $0.90(0.87 \text { to } 0.94)^{\star \star \star}$ \\
\hline MDSS score & & & & $0.94(0.89 \text { to } 1.00)^{*}$ \\
\hline Current smokers & & $3.46(1.54 \text { to } 7.81)^{\star \star}$ & $2.95(1.42 \text { to } 6.13)^{\star \star}$ & $5.24(1.76 \text { to } 15.65)^{\star \star}$ \\
\hline AUDIT-C score & $1.08(1.02 \text { to } 1.14)^{\star \star}$ & $1.26(1.18 \text { to } 1.35)^{\star \star *}$ & & $1.26(1.13 \text { to } 1.40)^{\star \star \star}$ \\
\hline PHQ-9 score & $1.10(1.06 \text { to } 1.13)^{\star \star \star}$ & $1.13(1.10 \text { to } 1.16)^{\star \star \star}$ & $1.15(1.10 \text { to } 1.21)^{\star \star \star}$ & $1.20(1.15 \text { to } 1.26)^{\star \star \star}$ \\
\hline
\end{tabular}

${ }^{*} \mathrm{P}<0.05 ;{ }^{* *} \mathrm{P}<0.01 ;{ }^{* * *} \mathrm{P}<0.001$.

†Inability to plan or look ahead.

łLack of perseverance and self-control.

§Novelty seeking and acting without thinking.

AUDIT-C, Alcohol Use Disorders Identification Test-Consumption; BIS-11, Barratt Impulsiveness Scale Version 11; DSH, deliberate self-harm; MDSS, Multidimensional Scale of Perceived Social Support; PHQ-9, Patient Health Questionnaire-9 item; RSES, Rosenberg Self-Esteem Scale; SA, suicidal attempt.

\section{DISCUSSION}

The findings of the current study show that the risk factors associated with DSH and SA were similar, but not identical, in our cohort of adolescent school students. The same risk factors for both DSH and SA included female gender, low school ranking, depression, substance use (tobacco and alcohol) and low self-esteem. These risk factors associated with DSH/SA can, therefore, be targeted in future prevention strategies. After adjusting for demographic factors and all other psychosocial factors, impulsivity was an independent risk factor for DSH but not for SA. However, factor 2 of the BIS (lack of perseverance and self-control) was significantly associated with SA in boys.

\section{Common risk factors}

The results of the current study with regards to gender differences in adolescent DSH/SA are consistent with previous findings, in that adolescent girls showed a higher prevalence of $\mathrm{DSH}^{528}$ and SA. ${ }^{729}{ }^{30}$ With respect

Table 5 Comparison of online assessments versus face-toface interviews of lifetime SA

\begin{tabular}{llccc}
\hline & & \multicolumn{3}{l}{ Interview assessment } \\
\cline { 3 - 5 } & & \multicolumn{2}{l}{ Lifetime } & Lifetime SA \\
& SA (-) & $(+)$ & Total \\
\hline \multirow{2}{*}{ Online } & Lifetime SA (-) & 89 & 9 & 98 \\
assessment & Lifetime SA (+) & 16 & 31 & 47 \\
& Total & 105 & 40 & 145 \\
\hline
\end{tabular}

SA, suicidal attempt. to the personality characteristics, low self-esteem has been associated with both $\mathrm{DSH}^{4}$ and SA. ${ }^{29}$ Cross-sectional surveys of adolescents have consistently found that depression is strongly correlated with $\mathrm{DSH}^{45}$ and SA. ${ }^{29} 30$ Tobacco smoking has also been previously identified to be a risk factor for $\mathrm{DSH}^{531}$ and $\mathrm{SA}^{32}{ }^{33}$ along with alcohol use for $\mathrm{DSH}^{52831}$ and $\mathrm{SA}^{3233}$ When we analysed the data according to gender, we found that tobacco smoking and alcohol use were especially important risk factors for DSH/SA in girls (tables 2 and 3). Compared with boys, the prevalence of smoking and alcohol use is relatively low in Taiwanese girls $(\mathrm{OR}=5.5$ and 5.8 , respectively, girls as the reference group).${ }^{34}$ Female smokers or alcohol users may be more pathological and have a lower threshold for DSH/SA. The risk factors in the current study are in good agreement with the existing literature, which underscores the need to offer preventive interventions by addressing each of these risk factors to reduce $\mathrm{DSH} / \mathrm{SA}$ and alleviate current distress. School surveys to identify adolescents with low self-esteem, depression or substance use are warranted, and mental health interventions for depression, substance use and to enhance self-esteem are also needed.

Stress due to school studies is usually high in Chinese society, and academic performance (ie, school ranking in this study) was a culturally specific factor for adolescent DSH and SA in this study. A previous study in Hong Kong reported that poor academic performance was related to adolescent non-suicidal self-harm and SA. ${ }^{35}$ Several explanations for the relationship between academic 
performance and DSH/SA have been proposed. Academic problems may precede DSH/SA, or they may both be caused by the same set of underlying risk factors. Students with poor academic performance should receive more attention from their teachers, because Chinese society emphasises academic performance and those with poor performance may have higher levels of frustration, poor self-esteem and hopelessness. Mental health and education policy-makers may need to provide low-ranking schools with more counselling services and support from mental health professionals; especially in girls' schools. Academic remediation activities may also be arranged to help students. Regular screening programme to early detect those who may need mental health services are also warranted. The education system and parents should also encourage other strengths rather than academic performance alone, such as studying at a vocational school. Further research should investigate the relationship between a student's academic high school performance and suicidality.

\section{Impulsivity}

There seems to be more evidence that impulsivity is associated with adolescent DSH than with SA. For example, previous studies in Europe and the USA have reported higher impulsiveness on self-reported scales as a risk factor for adolescent $\mathrm{DSH}^{4}{ }^{46}$ which is consistent with our findings. However, in contrast to previous studies, ${ }^{4}{ }^{42}$ our results do not support an association between overall impulsivity score and SA. Impulsivity could characterise the suicidal adolescents in models 1, 2 and 3, but it became non-significant after adding covariates of depressive symptoms (table 3 ). However, some studies have also reported no relationship between impulsivity and SA. In a case-control study comparing suicidal completers and community control adolescents, impulsivity was related to suicide in univariate analysis but not in multivariate analysis. ${ }^{37}$ In another study surveying suicidal and non-suicidal adolescent inpatients, the self-reported Impulse Control Scale score was not different between the two groups. ${ }^{38}$

When we focused on the moderating effects of gender, we found that total impulsivity score $(\mathrm{OR}=1.03)$ was correlated with DSH only in boys (model 6 of table 2). The OR was slightly higher compared with impulsivity among all students (OR=1.02, model 5 of table 2). Previous studies in Ireland also found that impulsivity was a risk factor for DSH only in boys. ${ }^{1539}$ Gender also modulated the association between impulsivity and SA (model 5 of table 3). However, impulsivity lost its significance in model 6 in both genders. We postulate that the moderating effect was weak.

Among the BIS subscales, BIS-11 factor 3 (novelty seeking and acting without thinking) was associated with DSH in both boys and girls in multivariate analysis (table 4). Inconsistent results regarding the relationship between impulsivity and DSH have been reported. Impulsivity, like suicidality, is a complicated construct consisting of many factors, rather than a unidimensional measure. ${ }^{40}$ Thus, the subscale analysis was warranted. Sensation seekers (ie, high factor 3 ) have been shown to pursue novelty even at the cost of self-harm, ${ }^{41}$ and this may explain our findings that factor 3 was correlated with DSH in both genders.

We also found that BIS-11 factor 2 (lack of perseverance and self-control) characterised SA in boys in multivariate analysis (table 4 ), which is consistent with prior studies. ${ }^{42} 43$ A previous Taiwanese study found that male adults with a history of SA exhibited higher BIS-11 factor 2 scores ${ }^{42}$ and Horesh et al also found an association between impulsivity and SA among male adolescent inpatients. ${ }^{43}$ A study using neuropsychological tests and brain imaging found that prepotent motor responses are more easily evoked in men, which is a tendency that could predispose them to impulse control disorders. ${ }^{44}$ In addition, suicidal male adolescents are more likely to exhibit an impulsive presentation than suicidal female adolescents. ${ }^{45}$ Since BIS-11 factor 2 is considered to reflect long-standing behavioural patterns ${ }^{46}$ it is plausible that boys with a lack of self-control and low self-esteem may be prone to suicidal behaviour. Gender modulated the association between impulsivity and SA and that may explain why BIS factor 2 score was significant in boys only.

Impulsive boys under stress such as those with depression or substance use may be less tolerant of complex thinking and lack of flexibility, and hence are predisposed to having a lower threshold for SA without contemplating the possible consequences. ${ }^{42} \mathrm{~A}$ neuroimaging study confirmed that BIS-11 subscales were correlated with different brain regions in adolescents. ${ }^{47}$ Another study of young adults found gender-specific differences in BIS-11Local Gyrification Index correlations in the middle and inferior frontal gyrus, ${ }^{48}$ which may explain our findings of a gender difference in impulsivity.

Our results may support the stress-diathesis theory that dispositionally impulsive adolescents and those with low self-esteem with mental illnesses such as depression are more likely to have SA. In a French study, impulsive traits of adolescents were related to the use of fewer appropriate and more inappropriate regulation strategies and depression. ${ }^{49}$ Psychological interventions to decrease impulsivity are also needed.

\section{Other psychosocial dimensions}

In hierarchical regression analysis of SA, social support was present in models 2 and 3, but disappeared after adjusting for substance use and depressive symptoms in model 4 (table 3). However, social support remained a risk factor for SA in the final model among girls. This may reflect that girls are affected by relationships with peers, family and teachers more than boys. A previous study also showed that social connectedness or family support ${ }^{30}$ was associated with adolescent suicidal risk. Social support in our study included that from family, peers and teachers, which may have contributed to the negative findings. Our results suggest that particular attention should be paid to high-risk girls with poor social support, and that active interventions should be arranged. 
Perceived family discord was a risk factor for DSH and SA in models 1, 2 and 3 of the current study, implying that family plays a role in adolescents' suicidality. This is consistent with previous $\mathrm{DSH}^{50}$ and SA studies. ${ }^{30}$ However, its effects on DSH and SA disappeared after adding depressive symptoms. A longitudinal study had similar findings in that after controlling for mental health disorders and adverse life events, the influence of parental and family factors on adolescent suicide was attenuated. ${ }^{51}$

\section{Strengths and limitations}

This study used a large community sample of adolescents from different areas of Taipei, which is a strength when it comes to generalisation. A further strength in the present study is that well-established self-reported measures and a broad array of relevant psychosocial measures were used. Finally, few studies not based in Western countries have investigated the risk factors for DSH and SA in a community sample.

There are also several limitations to this study. First, the overall response rate was only $60.61 \%$. Students in Taiwan have heavy study stress and that may hinder their motivation to participate. We worked together with school staff but we do not force students to participate in the study. Adolescents often are not interested in activities arranged by adults and that maybe one of the reasons that our response rate was not very high. We mentioned on the inform consents that we would select some students $(<10 \%)$ for diagnostic interview; maybe some students feel it is too time-consuming. Second, conducting a large-scale survey with such a large sample does not allow for individual in-depth interviews. Measures in the present study were self-reported, and thus may reflect bias. However, data from large school surveys by Hawton et al were also self-reported, ${ }^{14}$ and they found that the majority of respondents reporting lifetime DSH were also classified as having DSH by expert evaluation. ${ }^{52}$ This is similar to the findings of the current study in that the agreement between online assessments and interviews with regards to lifetime SA was moderate. In addition, we conducted the tests in a group setting, and the students could preview the online questionnaires, review and change their answers to decrease a social desirability effect and yield more reliable data. ${ }^{53}$ Self-reports using online questionnaires have been shown to allow students to report more sensitive risky behaviour than using paper-and-pencil questionnaires. ${ }^{54}$ Third, we did not use multiple assessments such as lab-measured impulsivity. However, a previous study showed a significant correlation between the self-reported form and the test of variables of attention measure of impulsivity $(\mathrm{r}=0.34 ; \mathrm{P}<0.05) .{ }^{38}$ Fourth, BIS-11 did not assess emotional impulsivity. However, one meta-analysis found that negative urgency, a form of emotional impulsivity, was significantly related to a composite of suicidality, and the effect size for negative urgency was larger than those reported for other forms of impulsivity. ${ }^{55}$ Emotional-relevant impulsivity needs to be assessed in future work. Fifth, this study was cross-sectional, precluding conclusions regarding causality. Future longitudinal studies examining alternative moderators are needed.

\section{CONCLUSIONS}

We found a number of similarities and differences in certain risk factors between adolescents with DSH and those with SA. Poor impulsivity and other factors such as female gender, low self-esteem, poor academic performance, depression and tobacco/alcohol use were significant risk factors in the final model of adolescent DSH. Gender modulated the relationship between impulsivity and DSH; associations between impulsivity and DSH were particularly strong among boys. Similar factors were associated with SA in the final model except for impulsivity, although its subscale was associated with SA in boys. Gender was also a moderator for the association between impulsivity and SA. These shared and unique risk factors are important to understand the mechanism behind DSH and SA, and to allow for incorporation of these risk factors into future research on prevention programme for these two behaviours among vulnerable adolescents across different settings.

Given the high prevalence rates and low consultation rates of these two behaviours, we suggest that these highrisk behaviours should be considered as a public health problem. Mental health professionals in schools should routinely assess DSH and SA, and self-reported screening questionnaires should be conducted along with routine physical examinations in school to help identify adolescents at risk. Paying attention to the mental health of adolescents at school may help to prevent the consequences associated with unidentified and untreated mental health problems.

\section{Author affiliations}

${ }^{1}$ Department of Medicine, MacKay Medical College, New Taipei City, Taiwan ${ }^{2}$ Department of Psychiatry, MacKay Memorial Hospital, Taipei, Taiwan ${ }^{3}$ Suicide Prevention Centre, MacKay Memorial Hospital, Taipei, Taiwan ${ }^{4}$ Mackay Junior College of Medicine, Nursing, and Management, Taipei, Taiwan ${ }^{5}$ Department of Psychiatry, En Chu Kong Hospital, New Taipei City, Taiwan ${ }^{6}$ Department of Psychiatry, College of Medicine, National Taiwan University, Taipei, Taiwan

${ }^{7}$ Department of Psychiatry, National Taiwan University Hospital, Taipei, Taiwan ${ }^{8}$ Department of Medical Research, MacKay Memorial Hospital, Taipei, Taiwan ${ }^{9}$ Department of Psychiatry, Taiwan Adventist Hospital, Taipei, Taiwan

${ }^{10}$ Department of Psychiatry, Cardinal Tien Hospital, New Taipei City, Taiwan

${ }^{11}$ Department of Psychiatry, Taipei City Psychiatric Center, Taipei City Hospital, Taipei, Taiwan

${ }^{12}$ Department of Electrical Engineering, National Taipei University of Technology, Taipei, Taiwan

Contributors Y-HH: contributed the data collection, performed the data analysis and drafted the article. H-CL: drafted the study protocol and helped in the data collection process. F-JT, K-YH and Y-HH: helped in the diagnostic interview process. F-JS: contributed in statistical analyses. Y-CC: contributed in counseling of at-risk adolescents, reviewing the article and revising it critically for important intellectual content. Y-PH: contributed in reviewing the article and revising it critically for important intellectual content. S-IL: monitored and guided the designing and implementation of the study, data collection, statistical analyses, interpreting findings and revising the article. All authors approved the manuscript submission for publication.

Funding Supported by grants from the Ministry of Science and Technology, Taiwan, Republic of China (NSC 9802314-B-195-011 MY3) and Mackay Memorial Hospital (9838, 9880), Taiwan.

Competing interests None declared. 
Patient consent Obtained.

Ethics approval Institutional Review Board of MacKay Memorial Hospital.

Provenance and peer review Not commissioned; externally peer reviewed.

Data sharing statement № additional data are available.

Open Access This is an Open Access article distributed in accordance with the Creative Commons Attribution Non Commercial (CC BY-NC 4.0) license, which permits others to distribute, remix, adapt, build upon this work non-commercially, and license their derivative works on different terms, provided the original work is properly cited and the use is non-commercial. See: http://creativecommons.org/ licenses/by-nc/4.0/

(c) Article author(s) (or their employer(s) unless otherwise stated in the text of the article) 2017. All rights reserved. No commercial use is permitted unless otherwise expressly granted.

\section{REFERENCES}

1. World Health Organisation. First WHO report on suicide prevention, 2014. http://www.who.int/mediacentre/news/releases/2014/suicideprevention-report/en/ (accessed 26 May 2017).

2. Owens D, Horrocks J, House A. Fatal and non-fatal repetition of selfharm. Systematic review. Br J Psychiatry 2002;181:193-9.

3. Hawton K, Bergen H, Casey D, et al. Self-harm in England: a tale of three cities. Multicentre study of self-harm. Soc Psychiatry Psychiatr Epidemiol 2007;42:513-21.

4. Madge N, Hawton K, McMahon EM, et al. Psychological characteristics, stressful life events and deliberate self-harm: findings from the Child \& Adolescent Self-harm in Europe (CASE) Study. Eur Child Adolesc Psychiatry 2011;20:499-508.

5. Moran P, Coffey C, Romaniuk H, et al. The natural history of selfharm from adolescence to young adulthood: a population-based cohort study. Lancet 2012;379:236-43.

6. Fergusson DM, Lynskey MT. Suicide attempts and suicidal ideation in a birth cohort of 16-year-old New Zealanders. J Am Acad Child Adolesc Psychiatry 1995;34:1308-17.

7. Andrews JA, Lewinsohn PM. Suicidal attempts among older adolescents: prevalence and co-occurrence with psychiatric disorders. J Am Acad Child Adolesc Psychiatry 1992;31:655-62.

8. Evans E, Hawton K, Rodham K, et al. The prevalence of suicidal phenomena in adolescents: a systematic review of population-based studies. Suicide Life Threat Behav 2005;35:239-50.

9. Lenkiewicz K, Racicka E, Bryńska A. Self-injury - placement in mental disorders classifications, risk factors and primary mechanisms. Review of the literature. Psychiatr Pol 2017;51:323-34.

10. van Heeringen K. The neurobiology of suicide and suicidality. Can J Psychiatry 2003;48:292-300.

11. Kim S, Lee KU. Research on potential biomarker correlates for suicidal behavior: A review. Asia Pac Psychiatry 2017:e12291.

12. Kashden J, Fremouw WJ, Callahan TS, et al. Impulsivity in suicidal and nonsuicidal adolescents. J Abnorm Child Psychol 1993;21:339-53.

13. Beautrais AL, Joyce PR, Mulder RT. Personality traits and cognitive styles as risk factors for serious suicide attempts among young people. Suicide Life Threat Behav 1999;29:37-47.

14. Hawton K, Rodham K, Evans E, et al. Deliberate self harm in adolescents: self report survey in schools in England. BMJ 2002;325:1207-11.

15. O'Connor RC, Rasmussen S, Hawton K. Adolescent selfharm: a school-based study in Northern Ireland. J Affect Disord 2014;159:46-52.

16. Huang $\mathrm{YH}$, Liu HC, Sun FJ, et al. Relationship Between Predictors of Incident Deliberate Self-Harm and Suicide Attempts Among Adolescents. J Adolesc Health 2017;60:612-8.

17. Winefield HR, Winefield AH, Tiggemann M. Social support and psychological well-being in young adults: the multi-dimensional support scale. J Pers Assess 1992;58:198-210.

18. Tsai FJ, Huang YH, Liu HC, et al. Patient health questionnaire for school-based depression screening among Chinese adolescents. Pediatrics 2014;133:e402-e409.

19. Li CS, Chen SH. Obsessive-compulsiveness and impulsivity in a non-clinical population of adolescent males and females. Psychiatry Res 2007;149:129-38.

20. Lin RC. Reliability and validity of the Rosenberg Self-esteem Scale on Chinese children. Journal of National Chung Cheng University 1990;1:29-46.

21. Patton JH, Stanford MS, Barratt ES. Factor structure of the Barratt impulsiveness scale. J Clin Psychol 1995;51:768-74.
22. Rosenberg M. Conceiving the self. New York: Basic Books, 1979.

23. Conigrave KM, Hall WD, Saunders JB. The AUDIT questionnaire: choosing a cut-off score. Alcohol Use Disorder Identification Test. Addiction 1995;90:1349-56.

24. Wu SI, Huang HC, Liu SI, et al. Validation and comparison of alcohol-screening instruments for identifying hazardous drinking in hospitalized patients in Taiwan. Alcohol Alcohol 2008;43:577-82.

25. Gau SF, Soong WT. Psychiatric comorbidity of adolescents with sleep terrors or sleepwalking: a case-control study. Aust N Z J Psychiatry 1999;33:734-9.

26. Baron RM, Kenny DA. The moderator-mediator variable distinction in social psychological research: conceptual, strategic, and statistical considerations. J Pers Soc Psychol 1986;51:1173-82.

27. Rosner B. Fundamentals of Biostatistics. Boston: Duxbruy, 2006.

28. Tsai $\mathrm{MH}$, Chen $\mathrm{YH}$, Chen $\mathrm{CD}$, et al. Deliberate self-harm by Taiwanese adolescents. Acta Paediatr 2011;100:e223-6.

29. Bridge JA, Goldstein TR, Brent DA. Adolescent suicide and suicidal behavior. J Child Psychol Psychiatry 2006;47:372-94.

30. Chen $\mathrm{CH}$, Chen $\mathrm{YH}$, Chen $\mathrm{CY}$, et al. Factors associated with suicide ideations and attempts in adolescents. Taiwanese J Psychiatry 2008;22:57-66.

31. Toprak S, Cetin I, Guven T, et al. Self-harm, suicidal ideation and suicide attempts among college students. Psychiatry Res 2011;187:140-4.

32. Kokkevi A, Richardson C, Olszewski D, et al. Multiple substance use and self-reported suicide attempts by adolescents in 16 European countries. Eur Child Adolesc Psychiatry 2012;21:443-50.

33. Wong SS, Zhou B, Goebert D, et al. The risk of adolescent suicide across patterns of drug use: a nationally representative study of high school students in the United States from 1999 to 2009. Soc Psychiatry Psychiatr Epidemiol 2013;48:1611-20.

34. Gau SS, Chong MY, Chen TH, et al. A 3-year panel study of mental disorders among adolescents in Taiwan. Am J Psychiatry 2005;162:1344-50.

35. Shek DTL, Yu L. Self-Harm and Suicidal Behaviors in Hong Kong Adolescents: Prevalence and Psychosocial Correlates. ScientificWorldJournal 2012;2012:1-14

36. Janis IB, Nock MK. Are self-injurers impulsive?: Results from two behavioral laboratory studies. Psychiatry Res 2009;169:261-7.

37. Renaud J, Berlim MT, McGirr A, et al. Current psychiatric morbidity, aggression/impulsivity, and personality dimensions in child and adolescent suicide: a case-control study. J Affect Disord 2008;105:221-8.

38. Horesh N. Self-report vs. computerized measures of impulsivity as a correlate of suicidal behavior. Crisis 2001;22:27-31.

39. McMahon EM, Reulbach U, Corcoran P, et al. Factors associated with deliberate self-harm among Irish adolescents. Psychol Med 2010;40:1811-9.

40. Wang YG, Chen S, Xu ZM, et al. Family history of suicide and high motor impulsivity distinguish suicide attempters from suicide ideators among college students. J Psychiatr Res 2017;90:21-5.

41. Mujica-Parodi LR, Carlson JM, Cha J, et al. The fine line between 'brave' and 'reckless': amygdala reactivity and regulation predict recognition of risk. Neuroimage 2014;103:1-9.

42. Wu CS, Liao SC, Lin KM, et al. Multidimensional assessments of impulsivity in subjects with history of suicidal attempts. Compr Psychiatry 2009;50:315-21.

43. Horesh N, Gothelf D, Ofek H, et al. Impulsivity as a correlate of suicidal behavior in adolescent psychiatric inpatients. Crisis 1999;20:8-14.

44. Li CS, Zhang S, Duann JR, et al. Gender Differences in Cognitive Control: an Extended Investigation of the Stop Signal Task. Brain Imaging Behav 2009;3:262-76.

45. Rice TR. Violence among young men: the importance of a genderspecific developmental approach to adolescent male suicide and homicide. Int J Adolesc Med Health 2015;27:177-81.

46. Moeller FG, Barratt ES, Dougherty DM, et al. Psychiatric aspects of impulsivity. Am J Psychiatry 2001;158:1783-93.

47. Fradkin Y, Khadka S, Bessette KL, et al. The relationship of impulsivity and cortical thickness in depressed and non-depressed adolescents. Brain Imaging Behav 2017;11:1515-25.

48. Hirjak D, Thomann AK, Kubera KM, et al. Cortical folding patterns are associated with impulsivity in healthy young adults. Brain Imaging Behav 2016.

49. d'Acremont M, Van der Linden M. How is impulsivity related to depression in adolescence? Evidence from a French validation of the cognitive emotion regulation questionnaire. $J$ Adolesc 2007;30:271-82

50. de Kloet L, Starling J, Hainsworth C, et al. Risk factors for self-harm in children and adolescents admitted to a mental health inpatient unit. Aust N Z J Psychiatry 2011;45:749-55. 
51. Fergusson DM, Woodward LJ, Horwood LJ. Risk factors and life processes associated with the onset of suicidal behaviour during adolescence and early adulthood. Psychol Med 2000;30:23-39.

52. Hawton K, Rodham K, Evans E. By their own young hand: Deliberate self-harm and suicidal ideas. London: Jessica Kingsley Publishers, 2006.

53. Whitener EM, Klein HJ. Equivalence of computerized and traditional research methods: The roles of scanning, social environment, and social desirability. Comput Human Behav 1995;11:65-75.
54. Booth-Kewley S, Larson GE, Miyoshi DK. Social desirability effects on computerized and paper-andpencil questionnaires. Comput Human Behav 2007;23:463-77.

55. Berg JM, Latzman RD, Bliwise NG, et al. Parsing the heterogeneity of impulsivity: A meta-analytic review of the behavioral implications of the UPPS for psychopathology. Psychol Assess 2015;27:1129-46. 